\title{
Design of Protocol Monitor Emulating Behaviors of TCP/IP Protocols
}

\author{
Toshihiko Kato, Tomohiko Ogishi, Akira Idoue and \\ Kenji Suzuki \\ $K D D R \& D$ Laboratories \\ 2-1-15, Ohara, Kamifukuoka-shi, Saitama 356, Japan \\ E-mail : \{kato, ogishi, idoue, suzuki\}@hsc.lab.kdd.co.jp
}

\begin{abstract}
Recently, the TCP/IP protocols are widely used and it is mentioned that, in some cases, throughput is limited due to problems such as network congestion. To solve such problems, the details of communication need to be examined. In order to support these examinations, we are developing an 'intelligent' protocol monitor which can estimate what communication has taken place by emulating the behaviors of the TCP protocol entities in a pair of communicating computers. This paper describes the overview of the monitor and the detailed design of the TCP behavior emulation function for both the state transition based behaviors and the internal procedures for the flow control, such as the slow start algorithm.
\end{abstract}

\section{Keywords}

Interoperability testing, protocol monitor, TCP/IP, TCP behavior emulation

\section{INTRODUCTION}

Recently, the TCP/IP protocols[1] are widely used in various computer 
communications. Here, most users of computers use communication functions installed in operating systems or commercial software products as they are, and do not pay attentions to their details. However, some problems occur for TCP/IP communications, in the cases that there are some packet losses due to network congestion and transmission errors, and that the protocol parameters of communicating computers are not matched. Especially, for TCP (Transmission Control Protocol), it is mentioned that the performance may be degraded due to the flow control mechanisms[1], and due to the use of send and receive socket buffers with different sizes[2]. When these problems occur, the details of communications need to be examined to detect the problem sources. For this purpose, it is common to use commercial protocol monitors[3]. However, these monitors have only the functions to capture PDUs (Protocol Data Units) transmitted over networks, to analyze their formats and parameter values, and to display the results. The analysis of packet sequences and the investigation of problem sources need to be performed manually by TCP experts.

In order to support of the analysis of details of computer communications, we have proposed an 'intelligent' protocol monitor which analyze protocol behaviors and detect protocol errors in communicating computers[4]. This monitor captures PDUs over networks, emulates protocol behaviors of communicating computers which send and receive the PDUs, and finds protocols errors if PDU formats and behaviors do not conform to the protocols. We have implemented the intelligent protocol monitor for OSI protocols[4].

By applying the technologies of the intelligent OSI protocol monitor to the TCP/IP protocols, we are currently developing a protocol monitor which emulates the behaviors of TCP/IP protocols. This monitor provides both the PDU monitoring function similar to conventional protocol monitors and the function which emulates the behaviors of a pair of communicating computers according to TCP. One of the largest differences between the TCP/IP protocols and the OSI protocols is that the modern TCP contains some internal procedures which are treated as "local matter" in the OSI protocols. These procedures include the slow start and congestion avoidance algorithms by which a sender controls the rate of injecting data segments into a network. As the results, the protocol emulation becomes much more complicated for TCP than for the corresponding protocol in the OSI protocol stack, i.e. OSI Transport Protocol class 4.

This paper describes the design of our protocol monitor which emulates TCP/IP protocol behaviors. The next section and section 3 describes the requirements and the overview of our TCP/IP protocol monitor, respectively. Section 4 describes the detailed design of the emulation function of TCP protocol. Section 5 gives some discusses on our monitor and section 6 makes some conclusions.

\section{REQUIREMENTS FOR TCP/IP PROTOCOL MONITOR}

We suppose the following requirements for our TCP/IP protocol monitor. 
(1) Since the TCP/IP protocols are used over high speed networks such as LANs, it is required that all PDUs transmitted over the network can be captured in an on-line operation, and that the monitoring results are examined by an operator in an off-line operation.

(2) As described above, the monitored TCP/IP communication needs to be examined in the following two points of view.

- Examining what PDUs are transmitted over the network which the monitor is attached to. This examination handles all of the PDUs captured by the monitor.

- Examining what communication has taken place between a specific pair of computers. Among the TCP/IP protocol suits, TCP has the most complicated protocol behaviors, and therefore this examination mainly focuses on TCP.

(3) In the latter case of (2), the behaviors of a computer for the captured TCP segment sequences are examined. In this examination, the state of TCP protocol entity in the computer is identified and its behaviors are emulated according to the TCP specification. We call this examination the TCP behavior emulation.

(4) As described above, modern TCP includes some internal procedures which are not specified in the state transition of the original TCP[5]. Furthermore, these procedures may not be implemented for some TCP/IP software products. The TCP behavior emulation needs to support these internal procedures and take account of the possibility that they are not used in the computers being examined.

(5) In some network configurations, LANs are interconnected via a WAN (wide area network) such as ISDN. In this case, there may be some time difference between the sending of PDUs by computers in the remote LANs and the capturing of them by the monitor. The TCP behavior emulation needs to take account of these time differences.

\section{OVERVIEW OF TCP/IP PROTOCOL MONITOR}

In order to satisfy the requirements described above, we have designed the following functions in our TCP/IP protocol monitor.

(1) As depicted in Fig. 1, the TCP/P protocol monitor is attached to a LAN and observes PDUs over the LAN. According to the requirements in Section 2, the monitor provides both the PDU monitoring function, which analyzes all TCP/IP PDUs transmitted over the LAN, and the TCP behavior emulation function focusing on the TCP protocol behavior of a specific pair of computers.

(2) The PDU monitoring function is similar to the function of conventional protocol monitors. It captures PDUs transmitted over the LAN, and analyzes PDU formats and parameter values according to TCP/IP protocols including 
ARP (Address Resolution Protocol), IP, ICMP (Internet Control Message Protocol), UDP (User Datagram Protocol) and TCP.

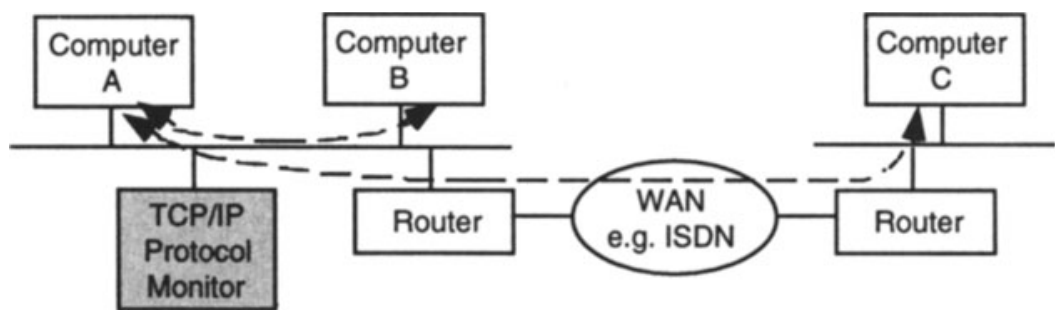

Figure 1 Example of network configuration with TCP/IP protocol monitor.

(3) The TCP behavior emulation function is realized by the following two steps.

First, the event sequence is estimated for an individual computer by taking account of the time differences between the PDU capturing by the monitor and the PDU handling in the computers being examined. These time differences may be negligible for the case that the computers are located in the same LAN (computers A and B in Fig. 1), but not negligible for the case that they are located in the remote LANs (computers $A$ and $C$ in Fig. 1).

Next, the behavior of the TCP protocol entity of an individual computer is emulated according to the estimated event sequence.

(4) The monitor function is implemented as software running in UNIX workstations. Figure 2 depicts the software structure of the monitor.

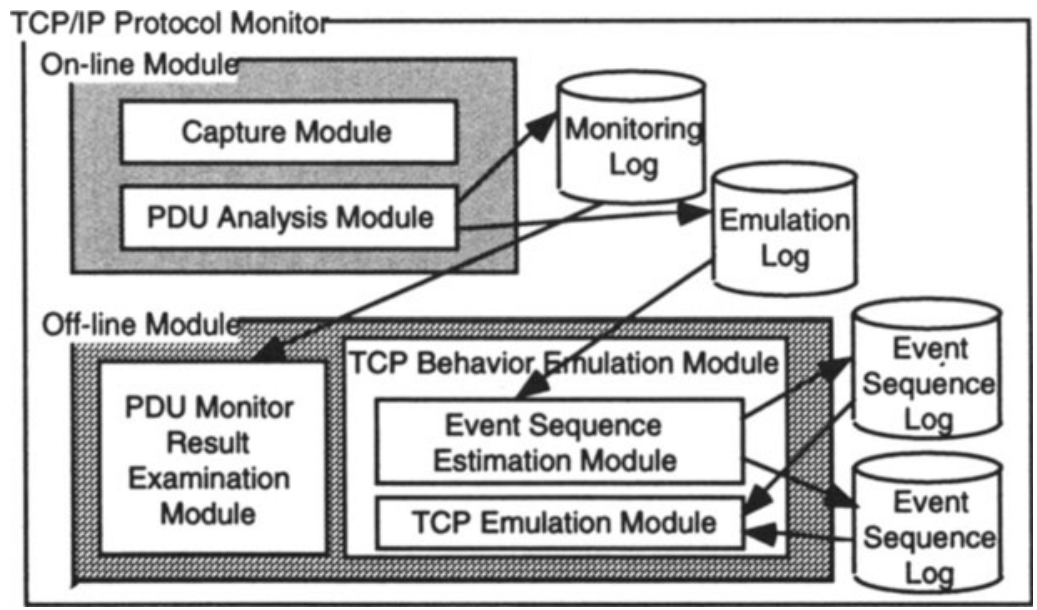

Figure 2 Software structure of monitor function.

It consists of an on-line and off-line module. The on-line module includes 
the capture module which captures PDUs transmitted over the LAN and the PDU analysis module which analyzes their format and parameter values according to the TCP/IP protocols. The PDU analysis module outputs the analysis results of the captured PDUs to the display of the workstation. This output is also saved in the monitoring log for the purpose of the off-line examination. It also saves the information used by the TCP behavior emulation in the emulation log.

(5) The off-line module consists of the PDU monitor result examination module and the TCP behavior emulation module. The PDU monitor result examination module allows an operator to examine the monitoring log by the help of editor functions such as cursor move and string search. The TCP behavior emulation module includes the event sequence estimation module and the TCP emulation module, which generate the event sequence for an individual computer and emulate TCP according to the estimated event sequence, respectively.

(6) The event sequence contains TCP protocol events, each of which is a "sent TCP segment" or a "received TCP segment", together with the estimated time of the event. The TCP emulation module maintains the state transition specification for TCP and processes each event according to the following procedure.

- When the event is a received TCP segment, it emulates the TCP behavior when the TCP protocol entity receives the segment. It looks up the corresponding state transitions and performs it. If it sends out a segment, the module checks a sent TCP segment in the event sequence and emulates the received and sent segments.

- When the event is a sent TCP segment, the protocol emulation module searches for the input which generates the segment, and it considers that the input is applied to the TCP protocol entity. If there are no input to generate the segment, it decides that the TCP protocol entity has some protocol errors.

\section{DETAILED DESIGN OF TCP BEHAVIOR EMULATION}

As described in the previous section, the TCP behavior emulation is realized by the emulation log generation by the on-line module, and the event sequence estimation and the TCP emulation by the off-line module. This section describes the details of these procedures.

\subsection{Generation of Emulation Log}

The PDU analysis module in the on-line module saves in the emulation log a record containing the following information for every captured TCP segment. This information is necessary to emulate the behavior the TCP protocol entity in computers. 
- The time when the beginning of a TCP segment is detected and the time when the end of a TCP segment is detected.

- The source and destination IP addresses.

- The parameters in TCP header except TCP checksum.

- The length of TCP segment including TCP header and TCP data.

- Whether TCP checksum is correct or not.

As for the time described above, the monitor software detects a PDU when the monitor has captured the whole data of the PDU. That is, the monitor software knows only the time when the end of a PDU is detected. The time for the beginning of a PDU is calculated from the length of the PDU and the transmission speed of the network.

In saving the above information, the procedure of IP, especially the reassembling of the fragmented IP datagrams is performed in the PDU analysis module. If a TCP segment is fragmented by IP, the following procedures are used.

- The length of TCP segment and whether TCP checksum is corrected or not are calculated after the reassembling.

- The time for the beginning of the TCP segment corresponds to that for the beginning of the first IP datagram containing the TCP segment. The time for the end of the TCP segment corresponds to that for the end of the last IP datagram containing the TCP segment.

\subsection{Estimation of Event Sequence}

The TCP behavior emulation module is invoked with a pair of IP addresses, which indicate the computers focused on. In the beginning of the TCP behavior emulation, the event sequence estimation module prepares an event sequence log for each computer from the emulation log. This is performed in the following way for computers $\mathrm{A}$ and $\mathrm{B}$.

(1) The module selects a record corresponding to a TCP segment without TCP checksum error exchanged between the specified computers.

(2) When a TCP segment is transferred from computer A to computer B, the module considers that an event of sent TCP segment takes place in computer $A$ and an event of received TCP segment in B.

(3) The module estimates the processing time of the event in computers A and B. For a sent TCP segment, the processing time is estimated as the time for the beginning of TCP segment minus the transmission delay between the monitor and the computer. For a received TCP segment, it is estimated as the time for the end of TCP segment plus the transmission delay. The transmissior delay is estimated by the propagation delay and the transmission time for a segment.

(4) The module reorders the records according to the estimated processing time. This reordering is performed independently for A and B. 
(5) By applying the procedures (1) through (4) to all records saved in the emulation log, the event sequence logs for A and B are generated.

Figure 3 shows an example of the event sequence estimation for two computers, $\mathrm{A}$ and $\mathrm{B}$, attached to LANs interconnected through ISDN with $64 \mathrm{Kbps}$ transmission speed and $100 \mathrm{msec}$ propagation delay. The TCP/IP protocol monitor captures some segments between computers $\mathrm{A}$ and $\mathrm{B}$, and generates the emulation $\log$ in the figure. As for computer $\mathrm{A}$, the delay with the monitor is negligible, the processing time in the event sequence $\log$ for $A$ is the same as either the beginning or end time in the emulation log.

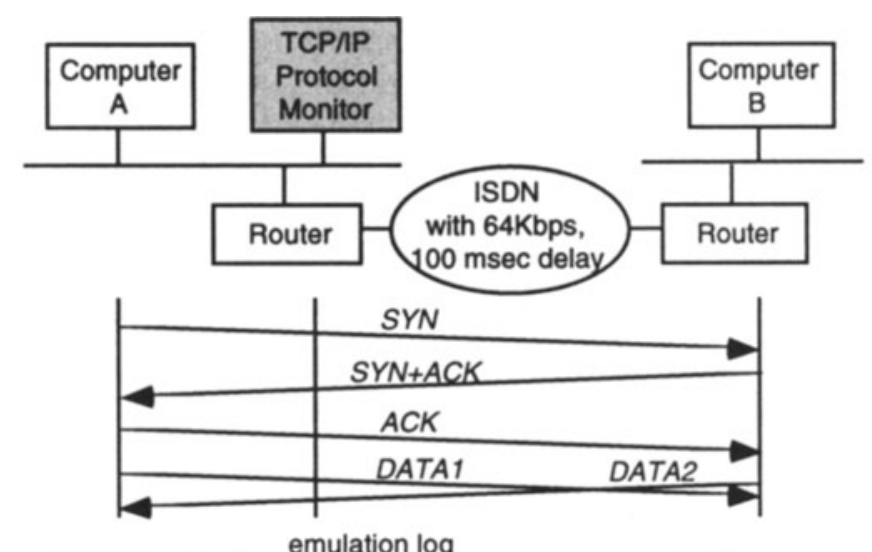

\begin{tabular}{|c|c|c|c|c|c|c|}
\hline type $s$ & source de: & begin & seg 1 & \multirow{2}{*}{$\frac{\text { seglen }}{24}$} & \multirow{6}{*}{\multicolumn{2}{|c|}{$\begin{array}{l}\text { Note: } 00: 00.123 \\
\text { indicate time whi } \\
\text { "mm:ss.msec". }\end{array}$}} \\
\hline$S Y N$ & A $B$ & $00: 00.123$ & $00: 00.123 \quad 24$ & & & \\
\hline \multicolumn{2}{|c|}{$S Y N+A C K$ B } & $00: 00.350$ & $00: 00.350$ & & & \\
\hline$A C K$ & A & $00: 00.353$ & $00: 00.353$ & & & \\
\hline DATA1 & A & $00: 01.455$ & $00: 01.456$ & & & \\
\hline DATA2 & B & $00: 01.566$ & 00:01.567 & & & \\
\hline \multicolumn{3}{|c|}{ event sequence $\log$ for $A$} & \multicolumn{4}{|c|}{ event sequence log for $\mathrm{B}$} \\
\hline pe & time & seglen & type & & me & seglen \\
\hline N sent & $00: 00.123$ & 24 & SYN recV & & 0.209 & 24 \\
\hline$A C K$ recv & $00: 00.350$ & 24 & $S Y N+A C K$ sent & & 0.246 & 24 \\
\hline$K$ sent & $00: 00.353$ & 20 & $A C K$ recv & & 0.458 & 20 \\
\hline F1 sent & $00: 01.455$ & 1480 & DATA2 sent & & 1.277 & 1480 \\
\hline IA2 recv & $00: 01.567$ & 1480 & DATA1 recv & & 1.745 & 1480 \\
\hline
\end{tabular}

Figure 3 Example of event sequence estimation.

On the other hand, the processing time in the event sequence $\log$ for $B$ is estimated from the propagation delay, $100 \mathrm{msec}$, and the transmission time of individual segments. For example, the processing time of DATA2 in computer B is estimated in the following way. 
- The length of DATA2 is 1500 byte including IP header, and it takes $1500 * 8$ $/ 64000=188 \mathrm{msec}$ to transmit DATA2 through ISDN. It takes $1 \mathrm{msec}$ to transmit it through the remote Ethernet.

- Therefore, the estimated processing time of DATA2 in computer B is given by the equation

00:01.566 -0.100 (propagation delay) $-0.188-0.001=00: 01.277$.

\subsection{Details of TCP Emulation}

By use of the event sequence logs, the TCP emulation module emulates the behavior of the TCP protocol entity of each computer. This module maintains the protocol behavior of TCP and traces how the entity behaves on an event by event basis. The behaviors are categorized into state transition based behavior and internal procedures of modern TCP. The rest of this section describes how these two kinds of behaviors are emulated by the TCP emulation module.

\section{Specification of State Transitions}

The TCP emulation module maintains the state and the internal variables to specify the state transition based behaviors for each TCP connection. The state takes the following values:

CLOSED, SYN_SENT, SYN_RCVD, ESTABLISHED,

FIN_WAIT_1 (state after the first FIN is sent),

FIN_WAIT_2 (state waiting for the second FIM),

CLOSING (state in simultaneous close),

CLOSE_WAIT (state waiting for close from the application after receiving FIN), and

LAST_ACK (state waiting for $A C K$ for $F I N$ ).

The internal variables maintained in the TCP emulation module include the followings:

send sequence variables such as

SND.NXT : the send sequence number to be sent next,

SND.UNA : the least send sequence number which is unacknowledged,

SND.WND : the maximum send sequence number which can be sent by the advertised window,

ISS : the initial send sequence number, and

MSS : the maximum segment size, and

receive sequence variables such as

RCV.NXT : the receive sequence number to be received next,

RCV.WND : the maximum receive sequence number which can be received by the advertised window, and

IRS : the initial receive sequence number. 
Figure 4 shows the state transitions of TCP. A state transition for one input and for one state is associated with one or more possibilities, each of which is specified by the condition, the output and the requirements for its parameters, the next state, and the variable update. The followings specify details for some state transitions in the figure.

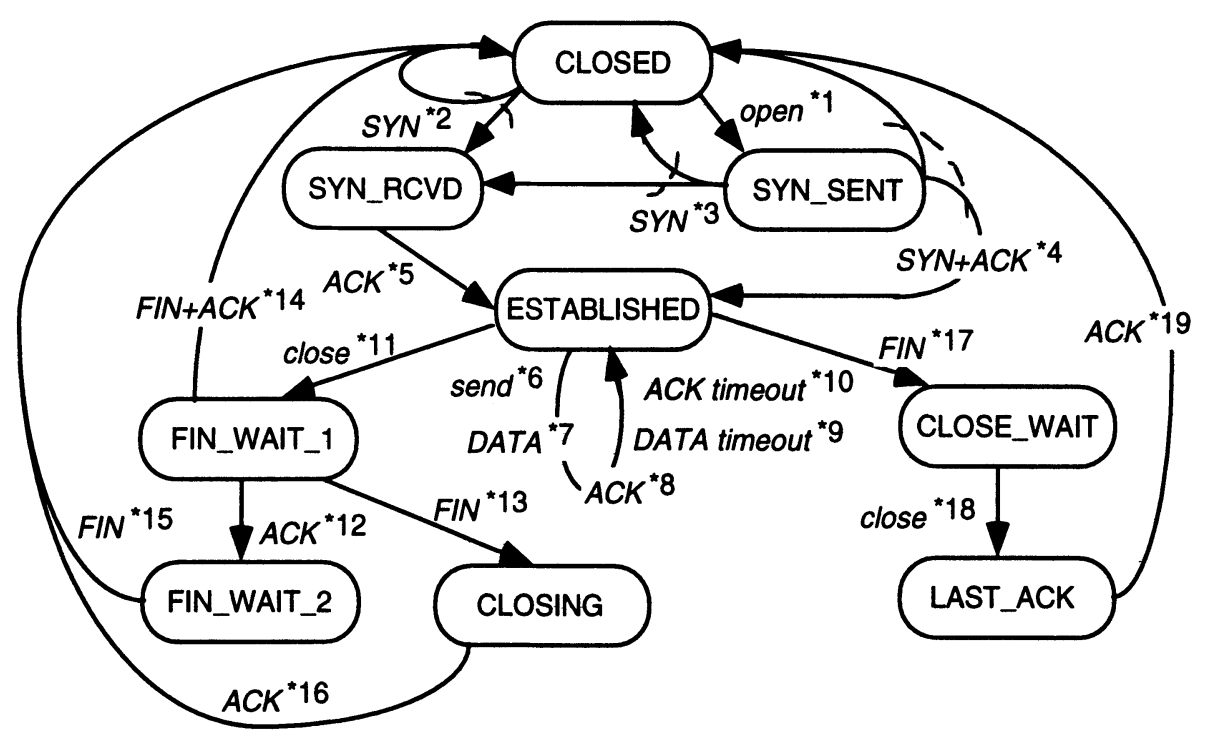

Figure 4 TCP state transition.

State Transition 1 : open in CLOSED

output : SYN; next state: SYN_SENT;

variable update:

ISS = sequence number (SEQ) in SYN; SND.UNA = ISS;

SND.NXT = ISS+1; SND.WND = window size (WND) in SYN;

MSS = maximum window size (MSS) in $S Y N$;

State Transition 2 : SYN in CLOSED

1) output: $S Y N+A C K$ with acknowledgment number (ACK)

$=$ SEQ in SYN+1; next state: SYN_RCVD;

variable update:

ISS = SEQ in SYN; IRS = SEQ in SYN+ACK; SND.UNA = ISS;

SND.NXT = ISS +1 ; SND.WND = WND in SYN; RCV.NXT = IRS +1 ;

RCV.WND = WND in $S Y N+A C K$;

MSS = min (MSS in SYN, MSS in SYN+ACK); or

2) output: $R S T+A C K$

with SEQ $=0$ and $\mathrm{ACK}=\mathrm{SEQ}$ in SYN + TCP data length (LEN) of SYN; next state: CLOSED; 
State Transition 4 : SYN+ACK in SYN_SENT

1) output: $A C K$ with SEQ = SND.NXT and ACK = SEQ in $S Y N+A C K+1$; next state: ESTABLISHED;

variable update:

IRS = SEQ in SYN+ACK; SND.UNA = ACK in SYN+ACK;

SND.WND = WND in SYN+ACK; RCV.NXT = IRS+1;

RCV.WND = WND in $A C K$;

MSS = min (current MSS, MSS in SYN+ACK); or

2) output: $R S T$ with $S E Q=A C K$ in $S Y N+A C K$; next state: CLOSED;

\section{Emulation Based on State Transition Specification}

By use of the state transitions defined in the previous section, the TCP emulation module traces the behaviors of TCP protocol entity. The algorithm is depicted in Fig. 5, and can be summarized as follows.

(1) The events saved in the event sequence log are traced one by one.

(2) If an event is a sent TCP segment (we call a sent event), the TCP emulation module searches for a transition for the current state which sends out the TCP segment. This is performed by looking up all of the transitions for the current state.

If such a transition exist, the TCP emulation module emulates the transition, including changing the state to the next state and updating the internal variables.

If such a transition does not exist, the TCP emulation module considers that there may be some protocol errors.

(3) If an event is a received TCP segment (we call a received event), the TCP emulation module looks up the state transition for the current state and the received segment. If the transition does not send out any outputs, then the transition is emulated.

If the transition sends out some outputs, the TCP emulation module looks for the next sent event in the event sequence log. If the next sent event is the correct output for the transition being traced, then the TCP emulation module reads out the sent event, and emulates the current received event and the sent event.

If the correct output is not found, the TCP emulation module supposes that there are no outputs for the event. If there is a possibility with no output for the transition, then the possibility is emulated. If there are no possibilities which do not send out any output for the transition, then the TCP emulation module considers that the current received event did not take place due to loss of the segment.

According to this algorithm, the behaviors of computer A in Fig. 3 are emulated in the following way. 


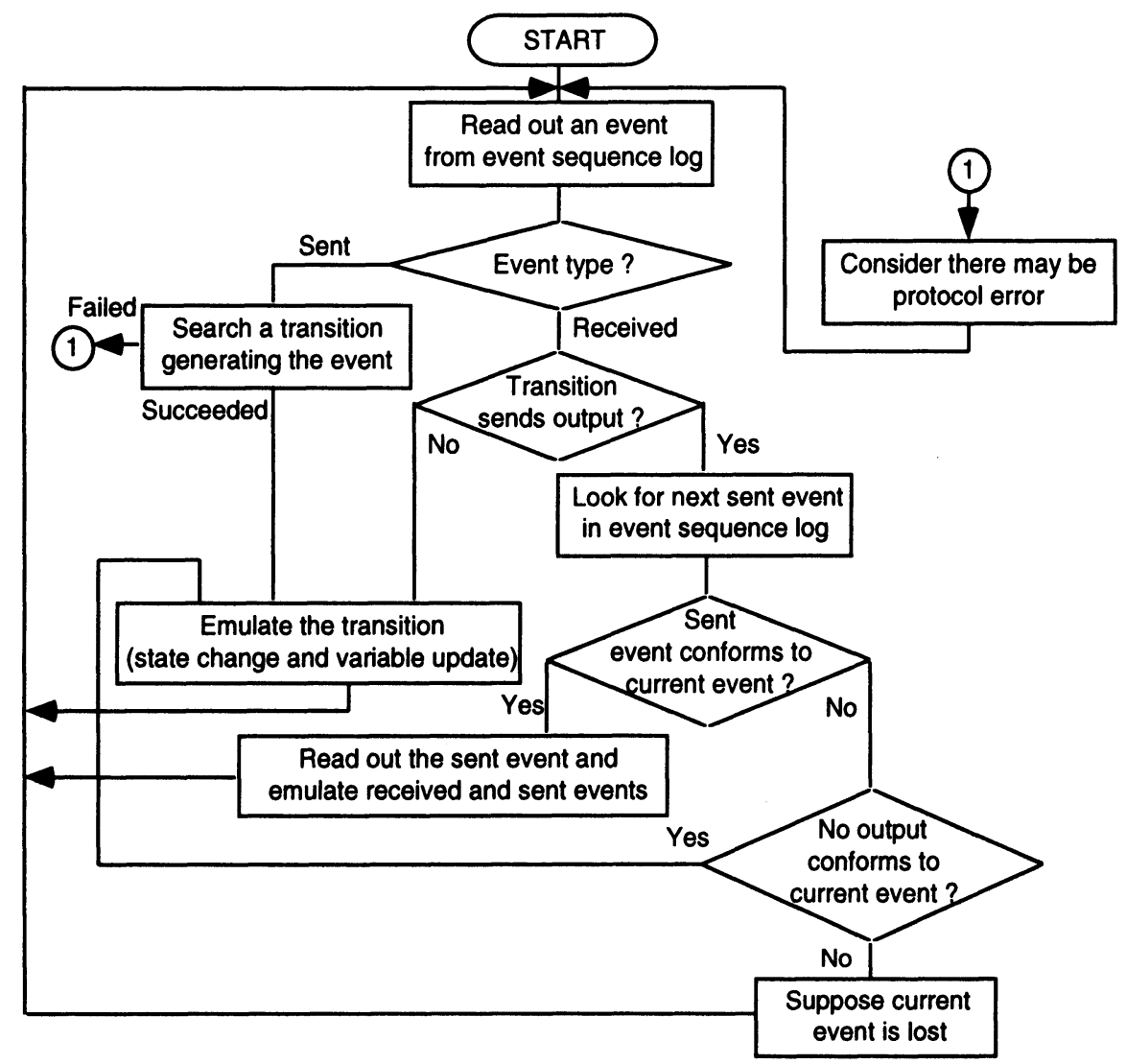

Figure 5 Algorithm for emulating state transitions.

- First, the TCP emulation module reads out an event 'SYN sent' and selects State Transition 1 for this event. The new state is SYN_SENT.

- Next, the module reads out an event ' $S Y N+A C K$ recv' and looks up State Transition 4. Since this transition includes some outputs, then the module looks for the next sent event in the event sequence log, and finds an event 'ACK sent'. This output conforms to possibility 1) in State Transition 4, ' $S Y N+A C K$ recV' and ' $A C K$ sent' are emulated. The new state is ESTBLISHED.

\section{Internal Procedures of Modern TCP}

Most of Modern TCP software products contain four flow control algorithms; slow start, congestion avoidance, fast retransmit, and fast recovery[1]. They are considered procedures defined internally by TCP protocol entities. 


\section{(1) Slow Start and Congestion Avoidance}

In Old TCP procedures, when a connection established, the sender injects multiple segments into the network, up to the window size advertised by the receiver. This may cause a problem that some segments are lost in routers if there is a slower link between the sender and receiver. Similarly, when any TCP segments are lost in the middle of communication, it is considered that there may be congestion some where in the network between the sender and receiver.

In order to solve this problem, the slow start and congestion algorithms are introduced. They use a congestion window, called cwnd, which control the sending rate internally in the sender, and a slow start threshold size, called ssthresh.

(1) Initialization for a given connection sets cwnd to one segment and ssthresh to 65535 byte.

(2) The sender never sends more than the minimum of cwnd and the advertised window from the receiver.

(3) When congestion occurs (indicated by a timeout or the reception of duplicate ACKs), one-half of the current window size (the minimum of cwnd and the advertised window) is saved in ssthresh. Additionally, if the congestion is indicated by a timeout, cwnd is set to one segment.

(4) When new data is acknowledged by the receiver, cwnd is increased in the following way. If cwnd is less than or equal to ssthresh, TCP is in slow start and cwnd is incremented by one segment every time an ACK is received. This opens the window exponentially. If cwnd is greater than ssthresh, congestion avoidance is being performed and the growth of cwnd is linear.

\section{(2) Fast Retransmit and Fast Recovery}

This algorithm allows TCP to retransmit a segment which is considered to be lost and, after that, to invoke congestion avoidance, not slow start. This algorithm may improve the throughput under moderate congestion, especially for large windows.

(1) When the sender receives three duplicate ACKs, it considers that these ACKs indicate that a segment has been lost. It sets ssthresh to one-half of the current cwnd and retransmits the missing segment.

(2) When an ACK arrives that acknowledges new data, the sender sets cwnd to ssthresh and starts the congestion avoidance.

\section{Emulation of Internal Procedures}

In order to emulate the internal procedures of TCP, we have adopted the following method.

(1) The TCP emulation module estimates the invocation of the internal procedures by monitoring the TCP segments. For example, it estimates that 
the slow start algorithm is invoked when a connection is established or when it detects a DATA segment retransmission caused by timeout (not duplicated ACKs). It also estimates the start of the fast retransmit when it detects a DATA segment retransmission invoked by three duplicated ACKs.

(2) The TCP emulation module maintains the following internal variables associated with the slow start and congestion avoidance algorithms and the fast retransmit and fast recovery algorithms:

variables associated with the slow start and congestion avoidance

CWND : the estimated congestion window,

SSTHRESH : the estimated slow start threshold, and

STATUS : indicates what algorithm is being emulated and takes NORMAL, SS (slow start), CA (congestion avoidance), FR (fast retransmit), and

variables associated with the fast retransmit and fast recovery

D_ACK : number of the received duplicated ACK.

(3) The TCP emulation module estimates the internal procedures by using these variables during the state transition emulation. The way of estimation is specified in the state transitions for send, DATA, ACK, and DATA timeout in state ESTABLISHED. The followings show examples.

State Transition 9 : DATA timeout in ESTABLISHED

output: DATA with SEQ in DATA != SND.NXT;

next state: ESTABLISHED;

variable update:

SSTHRESH $=\max (2 * M S S, 1 / 2 * \min (C W N D$, SND.WND $)$;

CWND = MSS; STATUS = SS;

In this state transition, the invocation of the slow start is detected and the interval variable SSTHRESH and CWND are estimated.

State Transition 6 : send in ESTABLISHED

output: DATA with SEQ in DATA >= SND.NXT;

next state: ESTABLISHED;

variable update:

SND.NXT = SEQ in DATA+LEN of DATA;

RCV.NXT = ACK in DATA; RCV.WND = WND in DATA;

if (SND.NXT - SND.UNA > CWND)

the internal procedures may not be used;

That is, whether the internal procedures are used or not is checked every time a DATA segment is transmitted.

State Transition 8 : ACK in ESTABLISHED

1) when D_ACK $==2$ and ACK in $A C K==$ SND.UNA

output: DATA with SEQ in DATA == SND.UNA;

next state: ESTABLISHED; 
variable update:

SSTHRESH $=\max (2 *$ MSS, $1 / 2 * \min (C W N D$, SND.WND $)$;

CWND = SSTHRESH+3*MSS; D_ACK $=0$; STATUS = FR;

/* This corresponds to the fast retransmit. */

2) when D_ACK $<2$ and ACK in $A C K==$ SND.UNA

next state: ESTABLISHED;

variable update:

if ( STATUS $!=F R$ ) D_ACK = D_ACK +1;

else CWND = CWND+MSS;

3) when SND.UNA < ACK in $A C K<$ SND.NXT

next state: ESTABLISHED;

variable update:

SND.UNA = ACK in ACK; SND.WND = WND in ACK; D_ACK =0;

if $($ STATUS $==$ FR $)$ CWND = SSTHRESH; STATUS = CA;

$I^{*}$ This is the start of congestion avoidance following the fast retransmit. */

$$
\begin{aligned}
& \text { if }(\text { STATUS }=\text { = SS }) \\
& \text { if }(\text { CWND }<=\text { SSTHRESH }) \text { CWND = CWND+MSS; } \\
& \text { else CWND = CWND + MSS } * \text { MSS / CWND; STATUS = CA; } \\
& \text { if ( STATUS = = CA ) } \\
& \text { CWND }=\text { CWND + MSS * MSS / CWND; } \\
& \text { if }(\text { CWND > 65535 ) STATUS = NORMAL; CWND = 65535; } \\
& / * \text { These two are the slow start and congestion avoidance } * /
\end{aligned}
$$

This transition specifies both the slow start and congestion avoidance algorithms and the fast retransmit and fast recovery algorithms.

(4) Since computers being examined may not support such internal procedures, the TCP emulation module will stop the emulation of those procedures when the monitored event sequences do not conform to the algorithms.

\section{DISCUSSIONS}

(1) It is considered that our TCP/IP protocol monitor is used effectively for the detailed analysis of TCP/IP communications. Especially, it is helpful to analyze the behavior of TCP internal procedures for the flow control. For example, our monitor can estimate the numbers of invocation of the slow start and the congestion avoidance algorithms. It can also estimate the number of DATA segment retransmission. These may help the detection of the problem sources of throughput degradation.

(2) We have the following comparisons with the development our intelligent protocol monitor for OSI protocols.

- The design of the protocol emulation function becomes simpler than that of our OSI protocol monitor, for the points that our monitor focuses only on the TCP emulation and that it performs the emulation in an off-line operation. 
- The design becomes more complicated for the points that our TCP/IP protocol monitor supports TCP internal procedures of the flow control. For this purpose, the TCP emulation module has introduced two state information, the state corresponding to the state transition based behavior, and internal variable STATUS which maintains what internal procedure is being emulated.

(3) Since TCP/IP is rather a mature protocol, it is considered that available protocol software does not include so many protocol errors. Our TCP/IP protocol monitor is used to analyze the details of communication, especially the details of TCP behaviors, such as the number of invocations of slow start.

(4) It is possible that our TCP/IP protocol monitor performs a wrong estimation of event sequence and a wrong TCP emulation. For example, the estimation of event does not take account of the buffering delay in routers. When such buffering delay is larger than propagation delay and transmission time, estimated event sequence by our monitor may be wrong in the actual processing order. In order to cope with such cases, it is required to reorder event sequence when any protocol error is detected during the emulation. It is possible to apply the rule based programming to implement such reordering based on heuristic algorithm[6].

\section{CONCLUSIONS}

In this paper, we have described the design of our TCP/IP protocol monitor which supports the detailed analysis of computer communications according to TCP/IP protocol. It provides the PDU monitoring function similar to conventional protocol monitors and, besides that, the function which can estimate what communication has taken place by emulating the behaviors of the TCP protocol entity in a pair of communicating computers. Since modern TCP includes some internal procedures for the flow control, such as the slow start algorithm, our monitor can emulate these procedures as well as the state transition based behaviors. This emulating functions are effective in analyzing TCP/IP communication, including counting how many times the slow start algorithm is invoked and how many DATA segments are retransmitted by the timeout retransmission and the fast retransmit algorithm.

\section{ACKNOWLEDGMENT}

The authors wish to thank Dr. H. Murakami, Director of KDD R\&D Laboratories, for his continuous encouragement to this study.

\section{REFERENCES}

[1] Stevens, W.R. (1994) TCP/IP Illustrated, Vol. 1 : The Protocols. Addison 
Wesley.

[2] Moldeklev, L. and Gunningberg, P. (1995) How a Large ATM MTU Causes

Deadlock in TCP Data Transfer. IEEE Trans. On Networking, 3.

[3] Tekelec (1992) Chameleon User's Manual.

[4] Ogishi, T., Idoue, A., Kato, T. and Suzuki, K. (1995) Design of Intelligent OSI

Protocol Monitor. in Proc. of the International Workshop on Protocol Test Systems, 351-66.

[5] DARPA Internet Program Protocol Specification (1981) Transmission Control Protocol. RFC 793.

[6] Kato, T., Ogishi, T., Idoue, A. and Suzuki, K. (1996) Design of Protocol Interoperability Testing System based on Rule Base Programming. in IPSJ SIG Notes, DPS 77-5. (in Japanese)

\section{BIOGRAPHY}

Toshihiko Kato is the senior manager of High Speed Communications Lab. in KDD R\&D Labs. Since joining KDD in 1983, he has been working in the field of OSI, formal specification and conformance testing, distributed processing, ATM and high speed protocols. He received the B.S., M.E. and Dr. Eng. Degree of electrical engineering from the University of Tokyo, in 1978, 1980 and 1983 respectively. Since 1993, he has been a Guest Associate Professor of Graduate School of Information Systems, in the University of Electro-Communications.

Tomohiko Ogishi is a member of High Speed Communications Lab. in KDD R\&D Labs. Since joining KDD in 1992, he worked in the field of computer communication. His current research interests include the protocol testing on TCP/IP communication. He received the B.S. Degree of electrical engineering from the University of Tokyo in 1992.

Akira Idoue is a research engineer of High Speed Communications Lab. In KDD R\&D Labs. Since joining KDD in 1986, he worked in the field of computer communication. His current research interests include implementation of high performance communication protocols and communication systems. He received the B.S. and M.E. Degree of electrical engineering from Kobe University, Kobe, Japan, in 1984 and 1986 respectively.

Kenji Suzuki is the senior manager of R\&D Planning Group in KDD R\&D Labs. Since joining KDD in 1976, he worked in the field of computer communication. He received the B.S., M.E. and Dr. Eng. Degree of electrical engineering from Waseda University, Tokyo, Japan, in 1969, 1972 and 1976 respectively. He received Maejima Award from Communications Association of Japan in 1988, Achievement Award from the Institute of Electronics, Information and Communication Engineers in 1993, and Commendation by the Minister of State for Science and Technology (Persons of scientific and technological research merit) in 1995. Since 1993, he has been a Guest Professor of Graduate School of Information Systems, in the University of Electro-Communications. 